\title{
Development and Utilization of a Somatic Cell Hybrid Mapping Panel to Assign Notl Linking Probes to the Long Arm of Human Chromosome 6
}

\author{
Eckart U. Meese, ${ }^{*}$ Colette M. Witkowski, † Huda Y. Zoghbi, ‡ Eric J. Stanbridge, $\S$ \\ PaUl S. Meltzer, ${ }^{*} \|$ AND Jeffrey M. Trent* ${ }^{*}, 1$ \\ Departments of *Radiation Oncology, "Pediatrics, and IHuman Genetics, University of Michigan Medical Center, MSRB\| C560B, 1150 \\ West Medical Center Drive, Ann Arbor, Michigan 48109; †Department of Radiation Oncology, University of Arizona College \\ of Medicine, 1501 North Campbell, Tucson, Arizona 85724; $¥$ Department of Pediatrics, Baylor College of Medicine, \\ One Baylor Plaza, Houston, Texas 77030; and §Department of Microbiology and Molecular Genetics, \\ CCM (Med Sciences 1, B210), University of California, Irvine, California 92717 \\ Received June 12, 1991
}

A somatic cell hybrid mapping panel that defines seven regions of the long arm and one region of the short arm of human chromosome 6 has been developed. Utilizing this panel, 17 NotI boundary clones from a NotI linking library were regionally assigned to the long arm of chromosome 6 . The majority of these clones (11) were found to localize within band regions 6q24-q27. The nonuniform distribution of NotI sites may indicate a cluster of HTF islands and likely represents a coincidence of coding sequences in this region of chromosome 6. Cross-hybridization of these linking clones to DNA from other species (zoo hlots) provides further evidence for transcribed sequences in 7 of the NotI clones. These NotI clones were also used to identify corresponding NotI fragments using pulsed-field gel electrophoresis, facilitating further physical mapping of this region. Finally, regional assignment of five polymorphic probes to the long arm of chromosome 6 is also presented. These hybrids and probes should facilitate the construction of a physical and genetic linkage map to assist in the identification of disease loci along chromosome 6. (c) 1992 Academic Press, Inc.

\section{INTRODUCTION}

The overwhelming majority of information on the genetic map of chromosome 6 relates to the study of the major histocompatibility complex (MHC) on the short arm, at 6p21.3. Even though chromosome 6 contains several important clinical markers, classic enzyme loci, protooncogenes, and approximately $5.8 \%$ of the human genome (approximately 175 million bp of DNA), surprisingly few genetic markers (outside the MHC) have been localized to this chromosome. One technical hindrance to probe localization on chromosome 6 has been a rela-

\footnotetext{
${ }^{1}$ To whom reprint request should be addressed at University of Michigan Medical Center, Department of Radiation Oncology, Division of Cancer Biology, 1150 W. Medical Center Dr., MSRBII, C560, Ann Arbor, MI 48109-0668.
}

tive paucity of hybrids with defined breakpoints along chromosome 6 (especially for the long arm), due in large part to the lack of a marker for direct selection on this chromosome.

Our interest in chromosome 6 stems from several lines of evidence suggesting the nonrandom rearrangement of the long arm in several malignant disorders (most notably malignant melanoma). In melanoma tumors, deletions or nonreciprocal translocations resulting in the loss of material from the long arm of chromosome 6 are observed in the majority of cases. At the molecular level, loss of heterozygosity for $6 \mathrm{q}$ has been documented (Millikin et al., 1991), and recently microcell-mediated chromosome transfer has provided biologic evidence for a tumor suppressor gene on human chromosome 6 (Trent et al., 1990). Hindering the identification of this putative tumor suppressor gene has been the lack of large numbers of regionally mapped molecular probes for 6q. Thus, as a first and fundamental step to facilitate further studies on chromosome 6 , we now report the development of a somatic cell hybrid mapping panel to define subregions of the long arm of chromosome 6.

This panel has been used to assign clones from a linking library consisting of genomic clones spanning the restriction site for the rare-cutting enzyme NotI. These NotI probes are of significant interest because of their facilitation in construction of long-range physical maps and, equally important, their possible utility in identifying additional coding sequences. Thus, the recognition that $\mathrm{CpG}$ islands often reside near the $5^{\prime}$ end of transcription units (and the preferential cutting of $\mathrm{CpG}$ by rare-cutting restriction enzymes like NotI) provides a means for identifying conserved and potentially transcribed sequences in a region of interest.

This report describes a detailed hybrid mapping panel that identifies eight distinct regions on chromosome 6 and provides specific information about the order of several DNA markers along the long arm. 


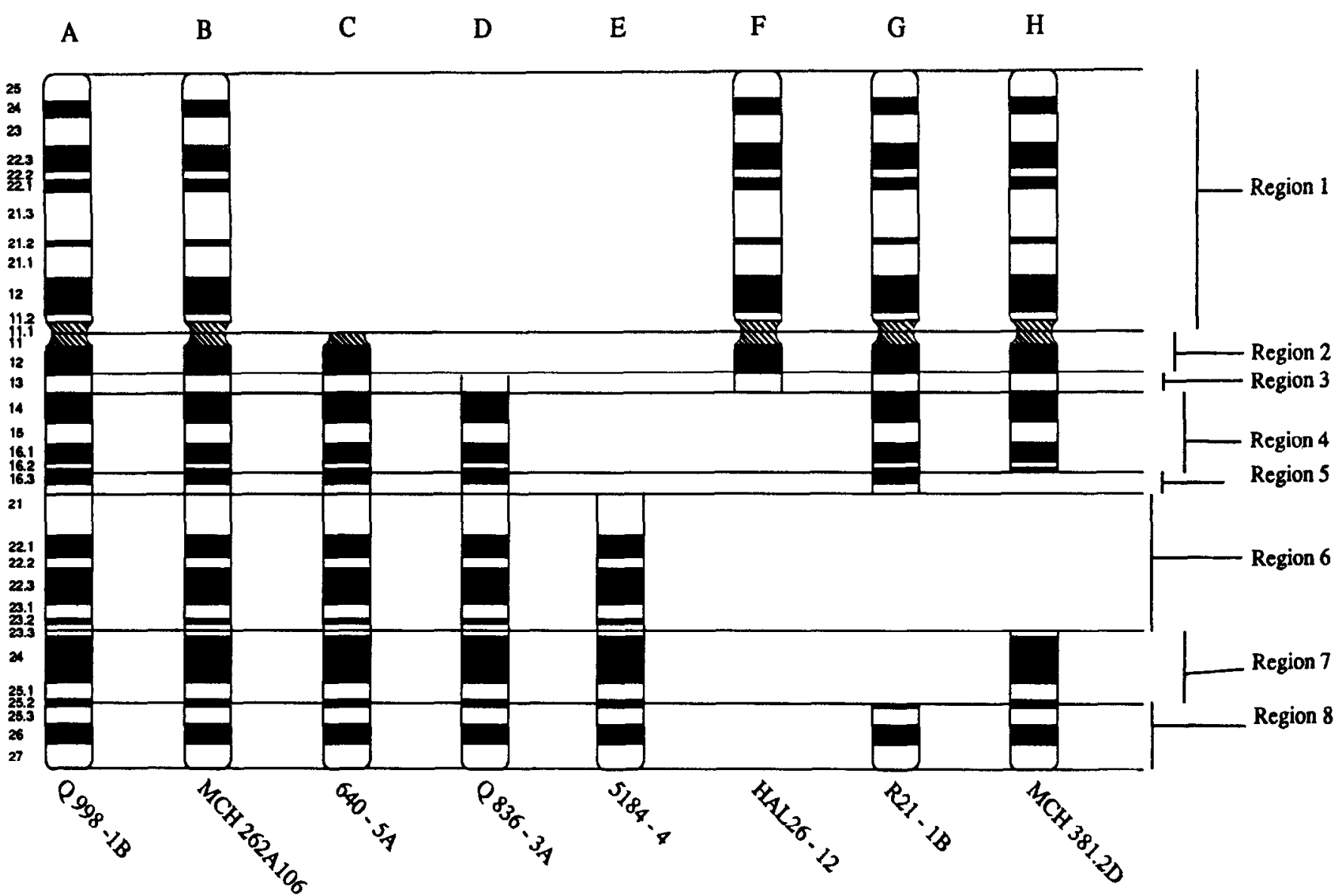

FIG. 1. Idiograms of the human chromosome 6-specific hybrid mapping panel. A description of the somatic cell hybrids is provided under Material and Methods. Each hybrid is referred to by a capital letter (A-H). To the right is a representation of the eight mapping regions delineated by these somatic cell hybrids.

\section{MATERIALS AND METHODS}

Preliminary results related to the mapping of eight NotI linking clones were presented in abstract form at HGM10 (Witkowski et al., 1989). Results presented here provide sublocalization of these probes, as well as our modification and significant expansion of this hybrid panel. The linking probes are referred to by their probe names. If locus symbols have been assigned, they are given in parentheses following the probe name.

Somatic cell hybrids. Hybrids R21-1B, 640-5A, Q998-1B, and 836$3 \mathrm{~A}$ were a generous gift from Dr. Carol Jones (Eleanor Roosevelt Institute, Denver, CO). Hybrid MCH262-A1D6 (generated by E. Stanbridge) was obtained following retroviral insertion of the $\mathrm{psv}_{2}$ neo gene into a normal chromosomal 6 followed by microcell-mediated chromosome transfer (Trent et al., 1990). MCH381.2D is a radiation reduction hybrid of MCH262-A1D6 generated by the method described by Dowdy et al. (1990).

Hybrids HAL26-12 and 5184-4 (generated by C. Witkowski) were produced by polyethylene glycol (PEG) fusion using a modification of the procedure of Davidson and Gerald (1976). Briefly, the thymidine kinase-deficient murine cell line clone-1D (a gift from D. H. Ledbetter, Baylor College of Medicine, Houston, TX) was fused to form mouse/human hybrids. Hybrids were selected in HAT medium, which contains hypoxanthine, aminopterin, and thymidine with quabain added to eliminate nonfused human cells. Mutant human cells for the 5184-4 hybrid were obtained from the NIGMS Human Genetic Mutant Cell Repository (GM05184) and were reported and confirmed to have a $t(6 ; 7)$ (q21;q21.2). Cells from the hybrid HAL26-12 were derived from a malignant melanoma cell line displaying a $t(1 ; 6)(q 21 ; q 13)$ (Trent et al, , 1989). A summary of the cell line designation, rodent background, chromosome 6 segment, and notation of other human chromosomes is provided in Table 1.
Chromosomal analysis. Chromosomes were harvested and Gbanded to identify breakpoints as previously described (Trent and Thompson, 1987). In addition to G-banding, documentation of human-specific sequences was obtained using G-11 staining (Bobrow and Cross, 1974). Documentation of chromosome 6-specific sequences was obtained by fluorescence in situ hybridization utilizing a painting probe specific for human chromosome 6 (obtained from Imaginetics, Naperville, IL). The protocol utilized for fluorescence in situ hybridization was modified from Pinkel et al. (1988). In addition, we generated chromosome 6-specific painting probes for the derivative chromosome 6 in two hybrids (R21-1B and MCH381.2D). They were generated by $E c o R I$ digestion of hybrid DNA followed by nick-translation using a biotin-labeled nucleotide.

DNA isolation, gel electrophoresis, and hybridization. DNA for standard Southern blotting was isolated from hybrid cell lines and various controls as previously described (Meese et al., 1989b). DNA was digested with the appropriate restriction enzyme and fractionated by electrophoresis on a $0.9 \%$ agarose gel. Prehybridization and hybridization were as described previously (Meese et al., 1989b). All probes were labeled using the random primer method of Feinberg and Vogelstein (1984).

Pulsed-field gel electrophoresis (PFGE) was performed using a modified CHEF system (Meese and Meltzer, 1990) and protocols previously described (Meese et al., 1989a).

NotI linking clones to chromosome 6. DNA was prepared in the laboratory of S. Weissman (Yale University) from a chromosome 6-specific library (LAO6NS01) by standard methods (Sambrook et al., 1989 ). Following digestion with $E c o R I$, the insert fragments were isolated on a low-melting-point agarose gel. The EcoRI fragments were shotgun-cloned into pUC19 and digested by NotI. Linearized fragments were recircularized in the presence of a kanamycin gene (1.9 kb) from Tn903, which was modified by the addition of NotI linkers. 


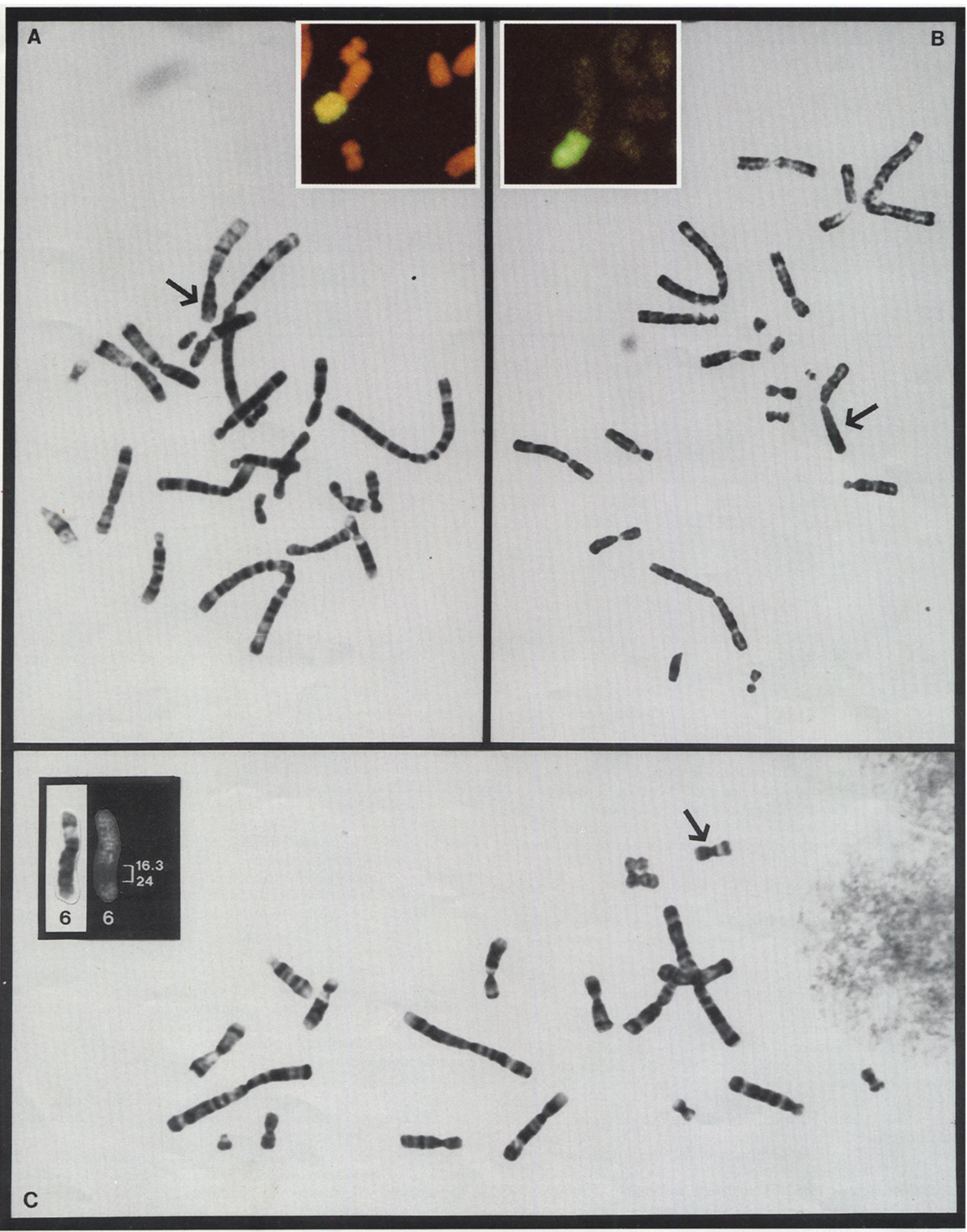


TABLE 1

\begin{tabular}{llll}
\hline \multicolumn{1}{c}{ Cell line } & Background & Chromosome 6 & $\begin{array}{c}\text { Other human } \\
\text { chromosome(s) }\end{array}$ \\
\hline Q998-1B & Hamster & Whole No. 6 & None \\
MCH262 AID6 & Mouse & Whole No. 6 & None \\
640-5A & Hamster & 6(cen-qter) & $9,10, \mathrm{Y}$ \\
Q836-3A & Hamster & 6(q13-qter) & Y \\
5184-4 & Mouse & 6(q21-qter) & 3, der7,10,15,17, \\
& & & $19,21,22$ \\
HAL26-12 & Mouse & 6(pter-q13) & $3,15,17,21$ \\
R21-1B & Hamster & del(6)(q21q25) & $6 \mathrm{q}-\mathrm{Y}$ \\
MCH381.2D & Mouse & del(6)(q16q24) & None \\
\hline
\end{tabular}

Colonies were selected for kanamycin resistance with the majority of the clones $(75 \%)$ documented to contain human DNA and to have the expected EcoRI insert in which the NotI kanamycin fragment was embedded.

Polymorphic probes for chromosome 6. The following chromosome loci were used: MYB, probe pHM2.6 (Dozier et al., 1986); D6S44, probe pYNZ132 (Nakamura et al., 1988); ESR, probe pOR3 (Gosden et al., 1986); D6S39, probe pTHH5 (O'Connell et al., 1987); and D6S29, probe pHHH157 (Hoff et al., 1988).

\section{RESULTS}

\section{Isolation and Characterization of Hybrid Cell Lines}

Figure 1 provides a schematic representation of the somatic cell hybrid mapping panel utilized in these studies. Idiograms documenting the region of chromosome 6 present in each hybrid are provided and delineate eight regions (one short arm, seven long arm). Representative metaphase chromosomes documenting the chromosome 6 alterations in selected hybrids are presented in Fig. 2.

Cytogenetic analysis was performed on all nine hybrids to confirm that they contained the derivative chromosome 6 . Hybrids were characterized using G-banding, G-11 staining, and chromosome-painting analysis using chromosome 6-specific probes and fluorescence in situ hybridization (Fig. 2). As documented in Table 1, three hybrids contained chromosome 6 as their only human DNA (Q998-1B, MCH262-A1D6, and MCH381.2D). The MCH262-A1D6 hybrid line containing a normal chromosome 6 is the parent of the MCH381.2D hybrid, which was generated by radiation reduction. As shown in Table 1, the five remaining hybrid lines contained a variety of human chromosomes in addition to chromosome 6 (most notably the $\mathrm{Y}$ chromosome in hybrid lines 640-5A, R21-1B, and Q836-3A). Finally, hybrid HAL2612 (containing the region 6pter-q13) is derived from the fusion of mouse clone $1 D$ cells with cells from a human malignant melanoma cell line containing a translocation of chromosome 6 [t(1;6)](q21;q13) (Trent et al., 1989)].

No inconsistencies between our cytogenetic results and our studies using chromosome 6-specific DNA markers have been detected. As indicated by the idiogram in Fig. 1, the hybrid panel allows mapping of DNA markers into eight distinct physical regions designated Region 1-8: Region 1 contains 6pter-p11; Region 2, 6q11-q12; Region 3, 6q13; Region 4, 6q14-q16.2; Region 5, 6q16.3; Region 6, 6q21-23.2; Region 7, 6q23.3-25.2; and Region 8,6q25.3-qter. As demonstrated below, this panel permits an accurate localization of DNA markers along chromosome 6q, again with no inconsistencies, and will be particularly useful for discriminating clones within band region $6 q 24-q$ ter.

\section{Regional Assignment of NotI Clones and Polymorphic DNA Markers along Chromosome 6}

Two different sources of DNA probes have been utilized for regional assignment in this study, with results presented in Fig. 3. First, three anonymous polymorphic markers were regionally assigned on chromosome 6 utilizing this panel: pHHH157 (D6S29), 6p; pYNZ132 (D6S44), 6q25.3-qter, and pTHH5 (D6S39), 6q25.3qter (the latter two previously assigned to p21-qter) (Fig. 3). Two other polymorphic probes (ESR and MYB) were mapped relative to this panel (ESR, 6q23.3-25.2; MYB, 6q21-23.2). These two assignments corroborate previous mapping data for these probes and further validate the consistency of the mapping panel.

A NotI partial EcoRI boundary library was constructed from a chromosome 6-specific ATCC library (LA06NS01). Twenty-five single-copy clones from the long arm of chromosome 6 were selected for regional localization with 8 being found to originate from identical NotI sites. Of the remaining 17 NotI probes, the insert size ranged from 3.5 to $6.8 \mathrm{~kb}$ (Fig. 4). As illustrated in Fig. 3, one linking clone mapped to Region 3, two linking clones to Region 4, three linking clones to Region 6, six linking clones to Region 7, and five linking clones to Region 8. The probes PN2-6 (D6S78), PN2-34 (D6S64), PN2-11 (D6S66), PN2-39 (D6S67), which were previously assigned to q22-qter, have been sublocalized to Region 7 (6q23.3-q25.2) by the extended mapping panel. The probe PN2-29 (D6S68), which has previously been assigned to $6 \mathrm{q} 21$, was also mapped to the band $6 \mathrm{q} 23.3-\mathrm{q} 25.2$.

The location of these NotI linking probes (summarized in Fig. 3) demonstrates the apparent nonuniform distribution of probes along this chromosome with an abundance of probes in the $6 \mathrm{q} 24-$ qter region.

FIG. 2. Representative G-banded chromosomes documenting derivative chromosome 6 (arrows) from three hybrid cell lines (640-5A (A); Q836-3A (B); and R21-1B (C). The insets to A and B depict fluorescence in situ hybridization (FISH) analysis using a chromosome 6 painting probe (see Materials and Methods). These FISH results corroborate the G-banding and document the translocation of chromosome 6 to rodent chromosomes in these two hybrid lines. The inset to $\mathrm{C}$ depicts FISH analysis to a normal chromosome 6 using for a probe biotinylated EcoRI-digested DNA from the R21-1B hybrid cell line. As can be observed, comparing the G-banded normal chromosome 6 (inset, left) to the same chromosome "painted" by labeled DNA from the R21-1B cell line readily identifies an interstitial deletion in this hybrid (see Fig. 1). This approach was also utilized to document the interstitial deletion of the derivative 6 chromosome within the hybrid line MCH381.2D (results not shown). 


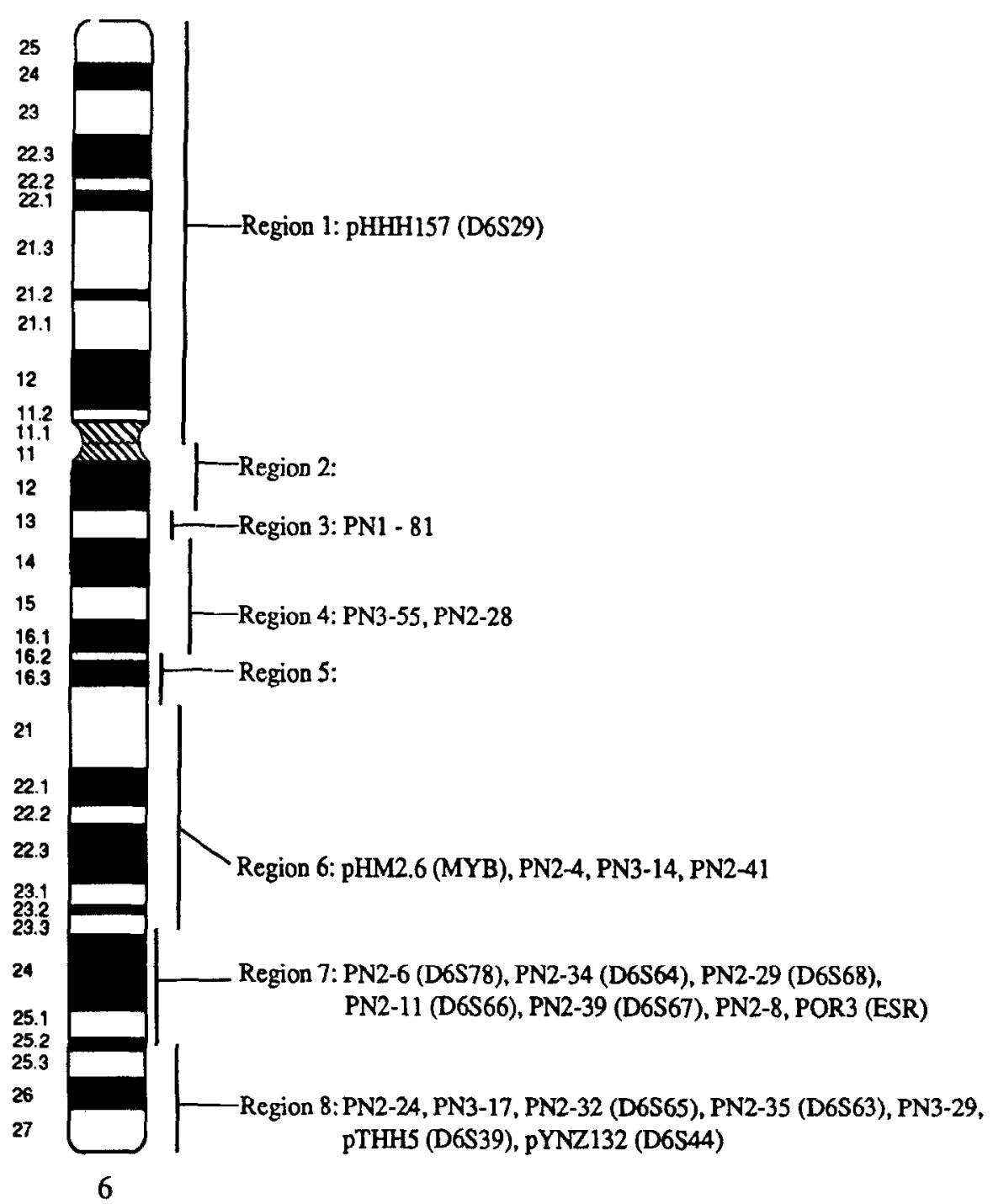

FIG. 3. Regional distribution of chromosome 6q probes. Seventeen NotI linking clones were mapped using the somatic cell hybrid panel shown in Fig. 1. In addition, six previously described polymorphic markers were sublocalized on 6q. If locus symbols have been assigned, they are given in parentheses following the probe name.

Importantly, to confirm further the validity of this panel, we remapped a subset of the Not I boundary clones using an independently derived somatic hybrid panel (Naylor et al., 1983). The clones we remapped included PN1-81, PN3-55, PN3-29, and PN3-14. In all cuses, the results of this panel (which is expanded for $6 p$ ) were in agreement with the localizations obtained by our $6 \mathrm{q}$ expanded mapping panel.

\section{PFGE Analysis of NotI Clones}

As described above, the nonuniform distribution of NotI linking clones along chromosome 6 may suggest the presence of CpG-enriched DNA (HTF islands) and raises the possibility of nearby transcribed regions. This association of transcribed regions within the NotI clones was examined by first cross-hybridizing $N o t I$ clones to DNA from different species (zoo blots). Of interest, 7/17 (41\%) of the NotI probes analyzed showed single-copy hybridization signals with mouse and hamster DNA suggestive of highly conserved sequences.

These NotI clones, in addition to providing an access to transcribed regions, can be used to establish largescale physical maps. Accordingly, each NotI clone can identify two adjacent NotI fragments, thereby greatly facilitating the construction of extended physical maps. As shown in Fig. 5A, clone PN2-24 identifies two NotI fragments on a PFGE gel that are adjacent to each other on chromosome 6. However, the majority of the NotI clones identified only one corresponding DNA fragment on a PFGE gel (Fig. 5B). To analyze NotI clones, we used PFGE conditions to separate DNA fragments up to 1500 $\mathrm{kb}$. Fragments larger than $1500 \mathrm{~kb}$ are not fractionated and remain in the wells of the gel or in the high-compression zone.

The results of the PFGE analysis demonstrate a different hybridization pattern for each NotI clone (results not shown). Since the smallest DNA fragment separated on PFGE was longer than $300 \mathrm{~kb}$, there must be a mini- 

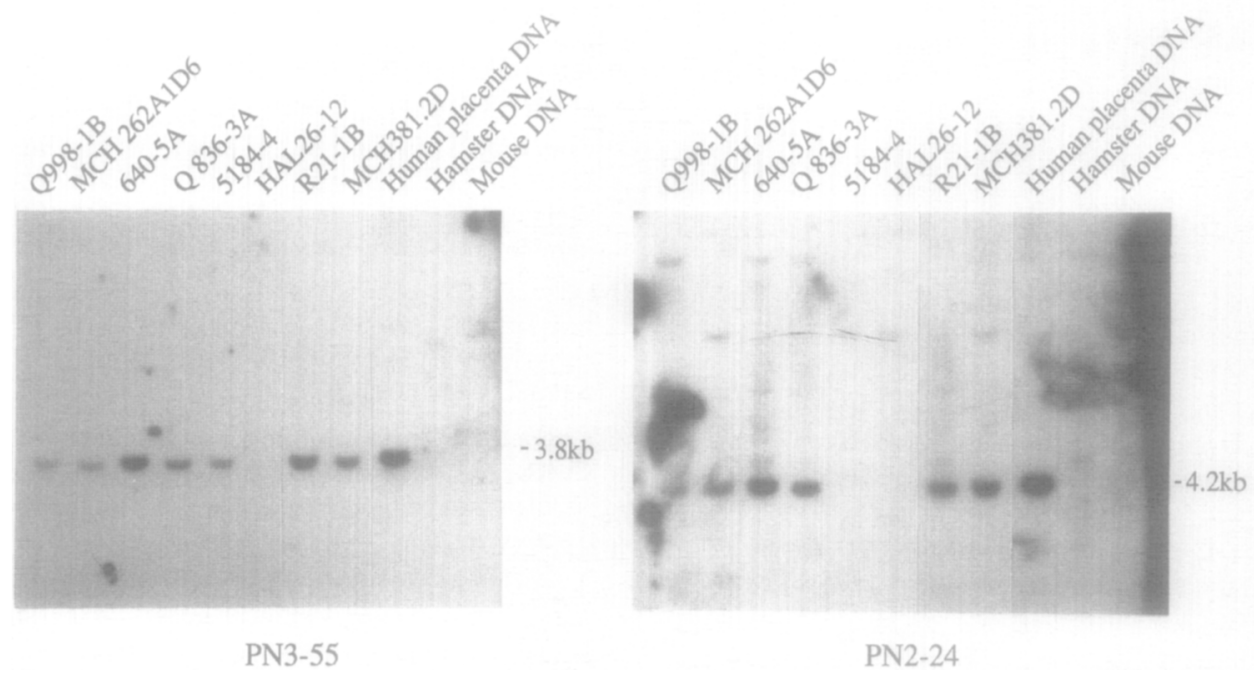

FIG. 4. Regional mapping of the probes PN3-55 and PN2-24 using the hybrid mapping panel shown in Fig. 1. DNA was isolated from the hybrids and digested with EcoRI.

mum distance of at least $300 \mathrm{~kb}$ between the different NotI clones analyzed. Thus, although several Not I sites have been found to be clustered in 6q24-qter, the NotI clones do not originate from identical sites within this chromosome band.

\section{DISCUSSION}

This report describes the assembly and development of a somatic cell hybrid mapping panel and the isolation and localization of polymorphic probes and NotI linking clones from chromosome 6 . The panel has been specifically developed to more accurately subdivide and extend mapping information around region 6q21-qter. This region has been documented to be the site of nonrandom chromosome alterations associated with human malignant melanoma, with recent biologic (Trent et al., 1990) and molecular studies (Meese et al., 1989b; Milliken et al., 1991) providing further evidence for the location of a putative tumor suppressor gene within this chromosomal region. Establishment of this collection of hybrids should greatly facilitate the ordering of probes within this region.

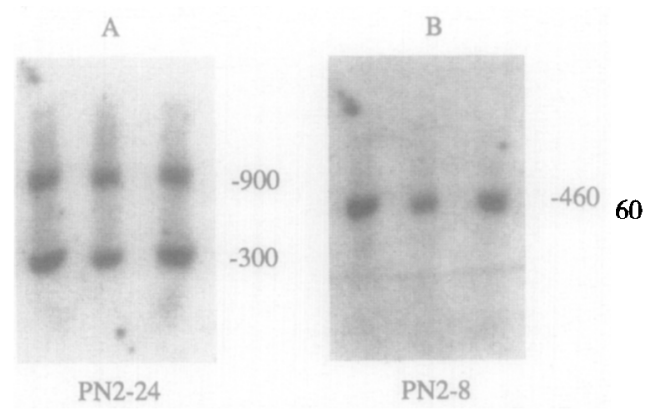

FIG. 5. Pulsed-field gels showing fragments detected by the probe PN2-24 (A) and probe PN2-8 (B). DNA from these different lymphocyte DNA samples was restricted by NotI and subsequently separated on PFGE using 180-s pulse time at $120 \mathrm{~V}$ for $36 \mathrm{~h}$. The molecular weight is given in $\mathbf{k b}$ (see text).
With regard to the regional assignment of NotI linking clones, the human genome is estimated to contain approximately 10,000 NotI restriction sites; thus, there are expected to be approximately 500 NotI sites on chromosome 6. To utilize these probes more efficiently for physical mapping, these NotI clones need to be localized further, and our hybrid panel offers a direct approach for regional assignment along the long arm of chromosome 6 .

Although preliminary, the results presented here provide evidence for the nonuniform distribution of NotI clones along chromosome 6, with the majority of NotI clones localizing within 6q24-qter. While these data are insufficient to address mechanisms for the nonuniform distribution, it appears likely that this library is overrepresented for clones within this region. The finding of nonuniformity of NotI libraries for this chromosome 6 library is similar to those previously described for several other chromosome-specific NotI libraries (e.g., Drabkin et al., 1990).

Finally, it is recognized that the construction of the complete physical and genetic map for a chromosome as large as chromosome 6 is a significant undertaking. However, the delineation of chromosomal regions along the long arm of this chromosome provided by our hybrid panel will simplify this task. The recognition of probes within specific regions should assist in the construction of both physical and genetic linkage maps, and it is hoped that this will facilitate identification of disease gene loci on chromosome $6 q$.

\section{ACKNOWLEDGMENTS}

We acknowledge Dr. Sherman Weissman and Dr. Iku Sawada (Yale University) for their generous provision of the NotI linking library and Dr. Carl-Heinz Grezschik for the gift of his previously developed hybrid panel. We also acknowledge the excellent technical assistance of Ms. Ann Burgess, Ms. Elizabeth Thompson, and Mr. Arthur Glatfelter. This research was supported in part by NIH Grants CA-29476 
(J.M.T.), CA19104 (E.J.S.), and by a grant from the Council for Tobacco Research-USA, Inc. (E.J.S.).

\section{REFERENCES}

Bobrow, M., and Cross, I. (1974). Differential staining of human and mouse chromosomes in interspecific cell hybrids. Nature 251: 77-79.

Davidson, R., and Gerald, P. (1976). Improved techniques for the induction of mammalian cell hybridization by polyethylene glycol. Somat. Cell Genet. 2: 165-176.

Dowdy, S., Scanlon, D., Fusching, C., Casey, L., and Stanbridge, E. (1990). Irradiation microcell-mediated chromosome transfer (XMMCT): The generation of specific chromosomal arm deletions. Genes Chrom. Cancer 2: 318-327.

Dozier, C., Walbaum, S., Leprince, D., and Stehelin, D. (1986). EcoRI KFLP linked to the human MYB gene. Nucleic Acids Res. 14: 1928.

Drabkin, H., Wright, M., Jonsen, M., Varkony, T., Jones, C., Sage, M., Gold, S., Morse, H., Mendez, M., and Erickson, P. (1990). Development of a somatic cell hybrid mapping panel and molecular probes for human chromosome 3. Genomics 8: 435-446.

Feinberg, A., and Vogelstein, B. (1984). Addendum: A technique for radiolabeling DNA restriction fragments to a high specific activity. Anal Biochem. 137: 66-67.

Gosden, J. R., Middleton, P. G., and Rout, D. (1986). Localization of the human estrogen receptor gene to chromosome 6q24-q27 by in situ hybridization. Cytogenet. Cell Genet. 43: 218-220.

Hoff, M., Nakamura, Y., Holm, T., Gillian, S., O'Connell, P., Leppert, M., Lathrop, G. M., Lalouel, J-M., and White, R. (1988). Isolation and mapping of a polymorphic DNA sequence ( $\mathrm{pHHH} 157$ ) on chromosome 6p (D6S29). Nucleic Acids Res. 16: 5217.

Meese, E., Meltzer, P. S., and Trent, J. M. (1989a). Application of natural partial digests to pulsed-field gel analysis of the amplified MDR locus. Genomics 5: 371-374.

Meese, E., Meltzer, P. S., Witkowski, C. M., and Trent, J. M. (1989b). Molecular mapping of the oncogene MYB and rearrangements in malignant melanoma. Genes, Chrom. Cancer 1: 88-94.

Meese, E., and Meltzer, P. (1990). A modified CHEF system for PFG analysis. Technique 2: 36-42.
Millikin, D., Meese, E., Vogelstein, B., Witkowski, C., and Trent, J. (1991). Loss of heterozygosity for loci on the long arm of chromosome 6 in human malignant melanoma. Cancer Res. 51: 5449-5453.

Nakamura, Y., Culver, M., Leppert, M., Sergeant, L., O'Connell, P., Lathrop, G. M., Lalouel, J-M., and White, R. (1988). Isolation and mapping of a polymorphic DNA sequence (pYNZ132) on chromosome 6 (D6S40). Nucleic Acids Res. 16: 5708.

Naylor, S., Sakaguchi, A., Shows, T., Grzeschick, C-H., Holmes, N., and Zastoff, M. (1983). Two nonallelic tRNA ${ }^{\text {met }}$ genes are located in the p23 $\rightarrow$ q12 region of human chromosome 6. Proc. Natl. Acad. Sci. USA 80: 5027-5031.

O'Connell, P., Nakamura, Y., Lathrop, G. M., Leppert, M., Cartwright, P., Lalouel, J-M., and White, R. (1987). Three genetic linkage groups on chromosome 8. Cytogenet. Cell Genet. 46: 673.

Pinkel, D., Landegent, J., Collins, C., Fuscoe, J., Segraves, R., Lucas, J., and Gray, J. (1988). Fluorescence in situ hybridization with human chromosome-specific libraries: Detection of trisomy 21 and translocations of chromosome 4. Proc. Natl. Acad. Sci. USA 85; 9138-9142.

Sambrook, J., Fritsch, E. F., and Maniatis, T. (1989). "Molecular Cloning: A Laboratory Manual," 2nd ed., Cold Spring Harbor Laboratory, Cold Spring Harbor, NY.

Trent, J., Stanbridge, E., McBride, H., Meese, E., Casey, G., Araujo, D., Witkowski, C., and Nagle, R. (1990). Tumorigenicity in human melanoma cell lines is controlled by introduction of human chromosome 6. Science 247: 568-571.

Trent, J. M., Thompson, F. H., and Meyskens, F. L. (1989). Identification of a recurring translocation site involving chromosome 6 in human malignant melanoma. Cancer Res. 49: 420-423.

Trent, J., and Thompson, F. (1987). Methods for chromosome banding of human and experimental tumors in vitro. In "Methods in Enzymology" (M. M. Gottesman, Ed.), Vol. 151, pp. 267-279, Academic Press, San Diego.

Witkowski, C., Zoghbi, H., Weissman, S., and Trent, J. (1989). As signment of Notl linking clones to the long arm of human chromosome 6 by a somatic cell hybrid mapping panel: Tenth Human Genome Mapping conference. Cylogervel. Cell Gertet 51: 1109. [Abstract] 